\title{
A Direct, Biomass-Based Synthesis of Benzoic Acid: Formic Acid- mediated Deoxygenation of the Glucose-Derived Materials Quinic Acid and Shikimic Acid ${ }^{\mathrm{a}} \uparrow$
}

\author{
Elena Arceo, Jonathan A. Ellman and Robert G. Bergman*b \\ 5 Received (in $X X X, X X X) X$ th $X X X X X X X X X 200 X$, Accepted Xth $X X X X X X X X X 200 X$ \\ First published on the web Xth $X X X X X X X X X 200 X$
}

An alternative biomass-based route to aromatics is described, in which quinic acid and shikimic acid, available from renewable 10 resources, are selectively converted to benzoic acid using a highly efficient formic acid-mediated deoxygenation method.

Today's chemical industry is highly dependent on crude oil as a feedstock to produce almost every commodity chemical or material. Aromatic hydrocarbons are among the most

15 important raw materials in the chemical industry and are obtained exclusively from fossil resources ${ }^{[1]}$. Production of industrial aromatic compounds from biomass resources could provide a sustainable alternative to traditional petroleum based manufacture and also eliminate the use of benzene-

20 based substances hazardous to human health and the environment.

Benzoic acid is prepared industrially by liquid-phase oxidation of toluene in the presence of cobalt catalysts (Dow, Snia Viscosa $)^{[2]}$. Benzoic acid is used as intermediate for the 25 manufacture of $\varepsilon$-caprolactam, terephthalic acid, dyes and perfumes and as a preservative in food, drugs and personal care products. In addition, oxidative decarboxylation of benzoic acid is an important route in the manufacturing of phenol $^{[3]}$, one of the largest volume chemicals derived from 30 benzene in the USA and Western Europe. Phenol is used mainly in the production of phenolic resins and bisphenol A for the manufacture of a wide variety of polymers and polymer additives.

biomass-based route

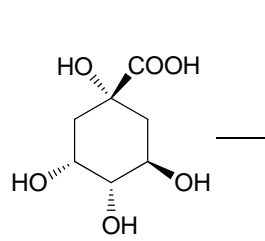

quinic acid

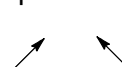
cinchona bark
coffee beans

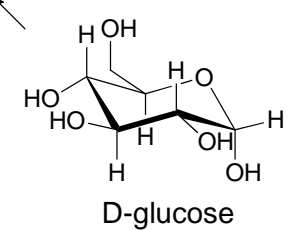

35

Scheme 1 Alternative Biomass-based Route to Benzoic acid

As part of an effort to develop new processes that support

the goals of sustainability, we describe herein an application of the formic acid-mediated didehydroxylation method ${ }^{[4]}$ to the preparation of benzoic acid from glucose derivatives that 40 eliminates petroleum based starting materials (Scheme 1). To the best of our knowledge there are no previous reports on the preparation of benzoic acid from non-aromatics.

$$
\text { D-(-)-Quinic acid } \quad((1 R, 3 R, 4 R, 5 R)-1,3,4,5 \text {-tetra- }
$$
hydroxycyclohexane-1-carboxylic acid) is a widely occurring 45 metabolite in plants and microorganisms, free or in the form of various esters with dihydroxycinnamic and gallic acid, known as chlorogenic acids. Quinic acid can be isolated from natural sources such as cinchona bark, coffee beans or tobacco, and recent advances in metabolic engineering have so developed cost effective environmentally friendly methods for the fermentative production of quinic acid from glucose $\mathrm{e}^{[5]}$. Processes for the conversion of quinic acid to hydroquinone ${ }^{[6]}$ and phenol ${ }^{[7]}$ have been reported, although in modest yields and selectivity.<smiles>O=C(O)[C@]1(O)C[C@@H](O)[C@H](O)C[C@H]1O</smiles>
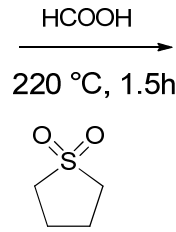

Scheme 2 Formic Acid-mediated Conversion of Quinic Acid to Benzoic Acid

The reaction of quinic acid and formic acid was first carried out using the experimental procedure previously ${ }_{60}$ reported for the synthesis of allyl alcohol from glycerol ${ }^{[4]}$, by heating a mixture of the starting polyhydroxylated carboxylic acid and formic acid at $230{ }^{\circ} \mathrm{C}$ in a distillation apparatus while directing a stream of nitrogen through the reaction mixture. However, in contrast to allyl alcohol, benzoic acid is a solid at ${ }_{65}$ room temperature, leading to complications when the reaction is run on a laboratory scale resulting from the sublimationdeposition of the final product throughout the distillation apparatus. In order to avoid this problem the use of a solvent was considered. Such a solvent must fulfill certain 70 characteristics to be optimal for the reaction: it should have a high boiling point, it should be thermally and chemically stable and it should be polar enough to solvate polyhydroxylated compounds. After screening several solvents such as triphosphates, glymes, diphenyl ether or 
chlorinated aromatics, sulfolane (tetramethylene sulfone) proved to be an excellent medium for the reaction. Sulfolane is a highly polar, thermally and chemically stable, watersoluble compound that is used industrially for the purification 5 of aromatic hydrocarbons and in several extractive distillations. ${ }^{[8-10]}$ Performing the reaction in sulfolane led to the formation and convenient isolation of benzoic acid in high yield and purity (Scheme 2).

In the formic acid-mediated didehydroxylation of glycerol, 10 the use of a nitrogen flow facilitated the distillation of allyl alcohol and was necessary to avoid charring of this sensitive alcohol. During the course of optimizing the reaction conditions for the synthesis of benzoic acid, we found that in this case an inert atmosphere was not required, undoubtedly 15 because both the starting material and the final product are highly stable at the reaction temperature.

The optimized reaction was performed using the following set-up and procedure: quinic acid $(10 \mathrm{mmol})$ was first dissolved in $10 \mathrm{~mL}$ of sulfolane at $30{ }^{\circ} \mathrm{C}$ in a two-necked flask 20 fitted with a fractioning column connected to a collecting flask through a u-shaped tube. The temperature in the reaction mixture was monitored by an immersed thermometer. Formic acid was then added ( 2 equivalents) and the mixture stirred at room temperature for about 15 minutes. The reaction mixture ${ }_{25}$ was then heated using a preheated sand bath and stirred at 220 ${ }^{\circ} \mathrm{C}$ for 30 minutes. After that time the procedure was repeated by adding a second portion of formic acid ( 2 equivalents). The reaction was monitored by removing aliquots and examining them by ${ }^{1} \mathrm{H}$ NMR spectrometry. After 45 minutes at the 30 indicated temperature, the conversion of quinic acid to benzoic acid was complete. In this process, water and some formic acid distilled from the reaction mixture and condensed in the collecting flask. Benzoic acid was extracted from the reaction mixture with diethyl ether (x6). The combined 35 ethereal phases were washed with cold water and dried over $\mathrm{MgSO}_{4}$ and the deoxygenated product was isolated by removing the solvent at reduced pressure. Spectroscopically pure benzoic acid was obtained in $92 \%$ yield without further purification.

40 The use of strong mineral acids, such as sulfuric acid combined with formic acid under various reaction conditions, did not result in significant yields of benzoic acid. Rather, unidentified decomposition products were observed, even when nitrogen gas was bubbled through the reaction mixture.

${ }_{45}$ Control experiments were performed without formic acid, in which quinic acid solutions in sulfolane were heated in the presence of other acids such as acetic acid, hydrochloric acid or sulfuric acid. The behavior of quinic acid in sulfolane at high temperatures, in the absence of any acid, was also 50 investigated. None of these experiments resulted in the formation of benzoic acid.

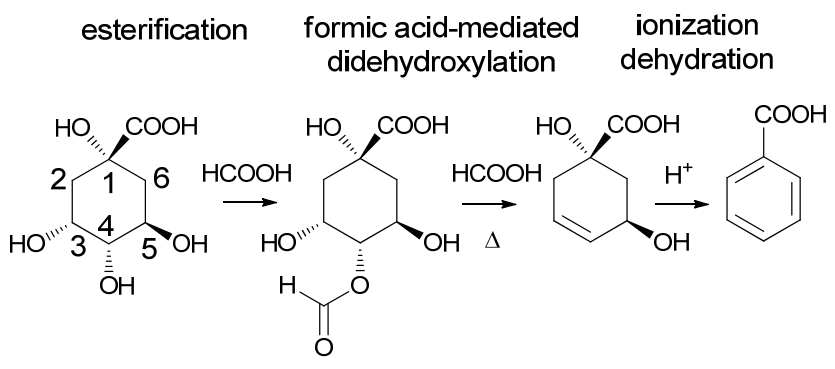

Scheme 3 Proposed reaction mechanism

55 The proposed reaction pathway for this transformation proceeds by didehydroxylation of the vicinal cis disposed hydroxy groups in positions 3 and 4 on the cycle. Consistent with the mechanism elucidated for the glycerol to allyl alcohol conversion, reversible esterification at either the $\mathrm{C} 3$ 60 or C4 hydroxy groups is followed by formation of the first unsaturated bond. Evolution to the conjugated aromatic system then occurs by subsequent acid-catalyzed dehydration (Scheme 3).<smiles>O=C(O)C1=C[C@@H](O)[C@H](O)[C@H](O)C1</smiles>

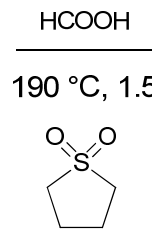<smiles>O=C(O)c1ccccc1</smiles>

Scheme 4 Formic Acid-mediated Conversion of Shikimic Acid to Benzoic Acid

Shikimic acid is also an important metabolite in aromatic amino acid biosynthesis. Although it is normally found in low concentrations in natural sources ${ }^{[11,12]}$, metabolic engineering 70 approaches have enabled fermentative production of shikimic acid from different carbon sources ${ }^{[13,14]}$. Gratifyingly, the deoxygenation of shikimic acid using the method described for quinic acid provided benzoic acid in a similar yield with complete selectivity. In this case the reaction temperature 75 could be lowered to $190{ }^{\circ} \mathrm{C}$ affording the product in $89 \%$ yield after extraction (Scheme 4).

\section{Conclusions}

In summary, a new synthetic connection has been made between renewable starting materials and aromatic chemicals 80 in which highly functionalized molecules derived from glucose are converted into a low oxygen-content and industrially useful compound using a simple, mild and onestep procedure that proceeds with high selectivity and efficiency. This provides encouragement for the development 85 of other "green" routes to useful aromatics from bioavailable polyhydroxy compounds.

\section{Notes and references}

${ }^{a}$ Department of Chemistry, University of California, Berkeley. Berkeley, CA 94720-1460 (USA); (J.A.E.) Fax: (+1) 510-642-8369; E-mail: 90 jellman@berkeley.edu 
${ }^{b}$ (R.G.B.)Department of Chemistry, University of California, Berkeley and Division of Chemical Sciences, Lawrence Berkeley National Laboratory, Berkeley, CA 94720-1460 (USA) Fax:(+1) 510-642-7714; E-mail: rbergman@berkeley.edu

$5 \uparrow$ This work was supported by the Dow Chemical Co. and the U.S. Department of Energy under Contract No. DE-AC02-05CH11231 (to R.G.B and J.A.E.).

1. K. Weissermel and H.-J. Arpe, in Industrial Organic Chemistry, Wiley-VCH, 2003, pp. 313-336.

2. K. Weissermel and H.-J. Arpe, in Industrial Organic Chemistry, Wiley-VCH, 2003, pp. 337-385.

3. W. Buijs, J. Mol. Catal. A: Chem., 1999, 146, 237-246.

4. E. Arceo, P. Marsden, R. G. Bergman and J. A. Ellman, Chem.

15 Commun., 2009, 3357-3359.

5. US Pat., 5798236, 1998.

6. N. Q. Ran, D. R. Knop, K. M. Draths and J. W. Frost, J. Am. Chem. Soc., 2001, 123, 10927-10934.

7. J. M. Gibson, P. S. Thomas, J. D. Thomas, J. L. Barker, S. S.

20 Chandran, M. K. Harrup, K. M. Draths and J. W. Frost, Angew. Chem., Int. Ed., 2001, 40, 1945-1948.

8. F. M. Lee and D. M. Coombs, Ind. Eng. Chem. Res., 1987, 26, 564573.

9. US Pat., 3466346,1969

25 10. US Pat., 5538599, 1996.

11. L. B. Enrich, M. L. Scheuermann, A. Mohadjer, K. R. Matthias, C. F. Eller, M. S. Newman, M. Fujinaka and T. Poon, Tetrahedron Lett., 2008, 49, 2503-2505.

12. R. H. Sui, Chem. Eng. Technol., 2008, 31, 469-473.

30 13. K. M. Draths, D. R. Knop and J. W. Frost, J. Am. Chem. Soc., 1999, 121, 1603-1604.

14. J. O. Ahn, H. W. Lee, R. Saha, M. S. Park, J. K. Jung and D. Y. Lee, J. Microbiol. Biotechnol., 2008, 18, 1773-1784. 